\title{
Flutter instability of freely hanging articulated pipes conveying fluid
}

\author{
Lionel Schouveiler* \\ Aix Marseille Univ, CNRS, Centrale Marseille, IRPHE, Marseille, France \\ Félix Chermette \\ Faculty of Engineering and the Environment, University of Southampton, Southampton, United Kingdom
}

\begin{abstract}
We experimentally investigate the stability of freely hanging articulated pipes made of rigid segments connected by flexible joints and with their displacements constrained in a vertical plane. When the velocity of the fluid conveyed by the pipe is increased, flutter-type instability occurs above a critical value. The critical velocity and the characteristics of the flutter modes (frequency, amplitude and shape) are determined as a function of the number $n$ of segments into the pipe which is varied from 2 to 5. Experimental results are compared to predictions from linear stability analysis extending previous studies by taking into account damping due to the dissipation in the joints. Qualitative agreement is found and the limits of the analysis are discussed.
\end{abstract}

* lionel.schouveiler@irphe.univ-mrs.fr 


\section{INTRODUCTION}

Dynamics of articulated pipes conveying fluid has been first studied by Benjamin [1, 2] as a discrete model of continuously flexible pipes. Benjamin [1] applied the Lagrangian method of analysis to a chain of $n$ rigid pipe segments, connected by flexible joints. The inlet position of the system is fixed while the outlet, where the flow discharges in the atmosphere, is free of displacement. This system is a discrete form, with a finite number of degrees of freedom, of the so called cantilevered flexible pipe. When, as considered by Benjamin, the articulated pipe motion is confined in a plane its dynamics is governed by a system of $n$ ordinary differential equations rather than a partial differential equation for its continuous counterpart. Then Benjamin focused on two-segment pipes, he analysed their linear stability [1] and conducted experiments [2]. He found both experimetally and theoretically that as the flow velocity is increased the static equilibrium position, corresponding to the aligned segments, can be destabilized at a critical value of the velocity. For a pipe freely hanging under gravity this instability manifests itself by an oscillatory motion of the pipe referred to as flutter and occuring through a Hopf bifurcation, or a static deflection, also called divergence, arising via a pitchfork bifurcation. In contrast, a horizontal pipe has been found to be subject only to flutter. Benjamin noted that the occurence of flutter, resulting from growth of small perturbations to attain limit cycle oscillations, requires a net transfer of energy from the fluid to the pipe during this phase. Later, Païdoussis et Deksnis [3] generalized the linear stability analysis of Benjamin to an arbitrary number of segments to study the transition, as $n$ is increased, between articulated pipe and continuous flexible pipe. They found that the critical velocities and frequencies for the flutter of articulated pipes converge to the values of the continuous system as $n$ is increased. These authors also showed that the critical velocity for buckling tends to infinity as $n \rightarrow \infty$, which could explained why freely hanging continuous pipes have been found to be only subject to flutter-type instability in a previous study of Païdoussis [4].

Rousselet and Herrmann [5] were the first to address nonlinear aspects of this system. For a two-segment hanging pipe whith zero stiffness joints, they analysed the stability of the limit cycles and deduced that the Hopf bifurcation changes from sub- to supercritical as the ratio of the fluid to the pipe mass is increased. In contrary to others, this study did not assume that the flow velocity is a prescribed parameter independent of time but, to take into account the nonstationarity induced by the pipe flutter, a flow equation was coupled to the equation of the pipe motion. We can also mention, among others, the study of Sethna and Shaw [6] which revealed a very complex phase portrait around the point in space of the system parameters where pitchfork and Hopf bifurcations occur simultaneously.

Modified forms of two-segment pipes have also been considered, some examples concerning freely hanging pipe configurations are presented. Bohn and Herrmann [7] analysed the linear stability of a hanging pipe when the two segments are allowed to oscillate in two different vertical planes and found a transition from loss of stability from equilibrium by flutter to loss by divergence as the angle between the two planes is incresased from 0 to $\pi / 2$. The effect of a harmonic component added to the mean flow has been analysed by Bohn and Herrmann [8], they studied the resonances with the natural frequencies of the system and their combinations and concluded that even a small oscillation amplitude of the flow rate has a destabilizing effect. Later Jensen [9] found with a analytical approach that a high frequency harmonic component, when compared to the natural frequencies of the system, could be either marginally destabilizing or stabilizing depending on the value of the ratio of fluid to pipe mass, he also reported a change in the nature of the Hopf bifurcation from super- to sub-critical as the amplitude of the harmonic component is increased.

Flow-induced vibrations of pipes conveying fluid in general, including the particular case of articulated pipes, have generated an abundant literature because of their evident practical interests. The bibliography presented here is not exhaustive, the reader can refer to the comprehensive review presented by Païdoussis [10]. The latter shows that the works devoted to articulated pipes have mainly focused on two-segment pipes and that the experimental results are fairly rare and incomplete. It should be pointed out that while the seminal works of Benjamin [1, 2] and the study of PaÏdoussis and Deksnis [3] were undertaken to deduce general statements about the stability of pipes conveying fluid, subsequent studies, including the present one, were mainly motivated by the fact the articulated pipes are examples of dynamical systems governed by ordinary differential equation system which can then be easily integrated and, moreover, that are amenable to experimental investigations. In the present study, we perfom experiments with articulated pipes made of 2 to 5 segments, our experimental setup is presented in §II. We also conduct a linear stability analysis accounting for the dissipation in the joints which was neglected in most of the previous studies, except for the references cited above in [7] and [9]. Details about this analysis are given in §III. Before conluding in $\S \mathrm{V}$, experimental and analytical results are presented together with comparisons in $\S \mathrm{IV}$. 


\section{EXPERIMENTAL SETUP}

A schematic of the experimental setup is shown in Fig. 1. An articulated pipe consists of $n$ identical rigid segments of length $L$, mass per unit length $M_{p}$, inner and outer radius $R_{i}$ and $R_{e}$, respectively. They are connected by $n-1$ identical flexible joints. The highest segment is connected, in $O$, by a first joint to a vertical pipe of same radii and fixed in the laboratory frame $(O, x, y, z)$. A water flow is supplied into the articulated pipe from its fixed top end $O$ and discharges into the atmosphere by its free end $E$. The volume-flow rate $Q$ is deduced from the mass-flow rate calculated by measuring the time to collect a set mass of water at the pipe exit, measured with a precision scale. The characteristic flow velocity is then calculated as $U=Q /\left(\pi R_{i}^{2}\right)$. With this method, the velocities $U$ are determined within a $5 \%$ uncertainty.

In order to constrain the motion of the pipe in the vertical plane $(O, x, z)$, two adjacent segments are connected with a pivot link of axis parallel to $(O, y)$. For this purpose, the segment ends are sharpened and attached together with a stainless steel axis inserted through holes drilled into the sharpened segment ends as shown in pictures of Fig. 1. This pivot link is then fitted with a short length of flexible silicon tube, of thickness 1 mm and inner radius equal to $R_{e}$, which is secured with four plastic collars. With this setup, the relative displacement of one segment relative to its neighbour is limited to about $\pi / 2$ well above the amplitude of the observed motions.

Experiments are performed changing the flow velocity $U$. At low $U$ the articulated pipe hangs statically under the action of gravity, whose acceleration is denoted by $\vec{G}$. At velocities above a critical value $U_{c}$ oscillatory displacement of the pipe in the vertical plane $(O, x, z)$ occurs. This planar motion is fully described by the $n$ angles $\phi_{r}$ between the $n$ segments and the vertical axis $(O, z)$. The pipe motion is recorded with a video camera operating at 10 images per second and placed perpendicular to the plane of the pipe motion. The length of the recorded sequences is of $100 \mathrm{~s}$. The $n$ angles $\phi_{r}$ and their temporal evolution are obtained by determinating from the successive images, the coordinates of two points of each segment axis, within a $2 \%$ uncertainty. Angular amplitude $\Phi_{r}$ and frequency $F_{r}$ can then be deduced by simple analysis of the temporal signals $\phi_{r}(T), r=1$ to $n$.

Present experiments are conducted with segments made of copper of $L=0.195 \mathrm{~m}, R_{e}=0.004 \mathrm{~m}, R_{i}=0.003 \mathrm{~m}$ giving $M_{p}=0.180 \mathrm{~kg} / \mathrm{m}$. It should be pointed out that $M_{p}$ is calculated dividing the total pipe mass by its total length, in such a way that it includes the mass of the fitting tubes, plastic collars and axes of the links. This means that the mass concentration at the joints due to these elements is neglected in the following and in particular in the analysis presented in the next section, this mass represents about $6 \%$ of the total mass of the articulated pipe. The conveyed fluid is water whose density is taken to be equal to $1000 \mathrm{~kg} / \mathrm{m}^{3}$ resulting in a mass of fluid contained by unit length of pipe $M_{f}=0.028 \mathrm{~kg} / \mathrm{m}$. The stiffness $K$ and damping $C$ coefficients of the joints (all supposed identical) are estimated considering the damped oscillator made of a single freely hanging segment, without fluid, while the upper segment is fixed vertically. The segment is slightly displaced from its equilibrium position then released. The damped oscillations are recorded with the camera from which we deduced $\phi(T)$ the temporal evolution of the angle made by the segment with the vertical axis. Estimations of $K$ and $C$ are then the values giving the best approximation of the small damped oscillations $\phi(T)$ by the expression of the linear damped oscillator which writes $\phi_{\text {lin }}(T)=\phi_{0} \cos \left(\Omega_{\text {lin }} T+\psi\right) \exp (-\mu T)$ where $\Omega_{\text {lin }}=\left(\frac{3 G}{2 L}+\frac{3 K}{M_{p} L^{3}}-\mu^{2}\right)^{1 / 2}$ is the pseudo-frequency and $\mu=\frac{3 C}{2 M_{p} L^{3}}$ the damping rate. Repeating this for 30 different joints, we get the mean values $K=0.235$ N.m and $C=0.00382 \mathrm{~N} . \mathrm{m} . \mathrm{s}$ with the fairly large standard deviations $\sigma_{K}=0.033$ N.m and $\sigma_{C}=0.00061$ N.m.s showing the difficulty of making identical joints.

\section{LINEAR STABILITY ANALYSIS}

To analyse the linear stability of the static equilibrium position of the hanging articulated pipe, we first derive the equations of motion by means of the Lagrange's formalism using the $n$ angles $\phi_{r}$ as generalized coordinates. As shown by Benjamin [1], the $n$ Lagrange's equations, for the open system composed of the $n$ segments and $n$ joints of the articulated pipe and of the fluid it contains at any times, write

$$
\frac{\mathrm{d}}{\mathrm{d} T} \frac{\partial \mathcal{L}}{\partial \dot{\phi}_{r}}-\frac{\partial \mathcal{L}}{\partial \phi_{r}}+\frac{\partial \mathcal{R}}{\partial \dot{\phi}_{r}}=-M_{f} U\left(\overrightarrow{\overrightarrow{O E}}+U \overrightarrow{\tau_{n}}\right) \cdot \frac{\partial \overrightarrow{O E}}{\partial \phi_{r}} \quad(r=1 . . n)
$$

where overdot denotes derivative with respect to time $T$.

$\mathcal{L}=\mathcal{T}-\mathcal{V}$ is the system Lagrangian, $\mathcal{T}=\mathcal{T}_{\text {pipe }}+\mathcal{T}_{\text {fluid }}$ is the sum of the kinetic energies of the pipe and of the fluid. $\mathcal{V}=\mathcal{V}_{\text {gravity }}+\mathcal{V}_{\text {restor }}$ is the potential energy accounting for the gravity force on the pipe and on the fluid, $\mathcal{V}_{\text {restor }}$ is associated to the restoring forces due to the stiffness of the joints. $\overrightarrow{\tau_{r}}$ is the unit vector parallel to the segment $r$ and then $\overrightarrow{O E}+U \overrightarrow{\tau_{n}}$ is the fluid velocity at the pipe free end. 


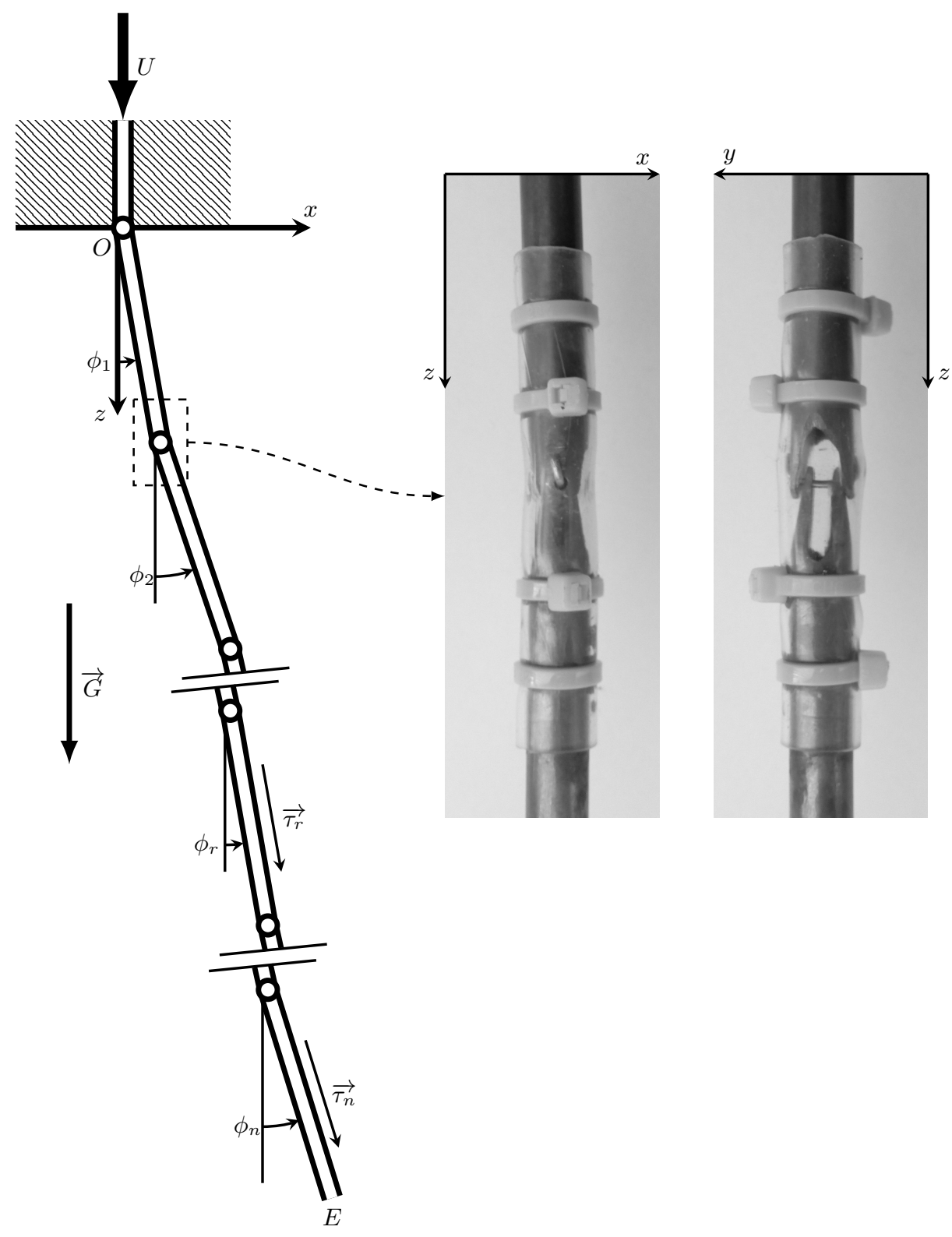

FIG. 1. Schematic of the experimental setup, pictures show two perpendicular views of a segment joint.

For the calculation of the lagrangian we consider for simplicity the radii $R_{e}$ and $R_{i}$ as being small compared to the segment length $L$. The flow is supposed to be incompressible and normal to any section of the pipe. Moreover, because the present analysis is undertaken to investigate the characteristic of the system at the flutter threshold, the flow rate is assumed time independent and the velocity profile uniform in any section. This later assumption is supported by the fact that, at the threshold, the pipe Reynolds number is larger than $5 \times 10^{4}$ corresponding to a fairly flat turbulent velocity profile. With these assumptions, in any section of the segment $r$ the flow velocity relative to this pipe segment writes $U \overrightarrow{\tau_{r}}$ where $U$ is constant. The restoring forces due to the joint stiffness are assumed linearly dependent on the deformation angle and characterized by the stiffness coefficient $K$.

With these assumptions, the calculation of the lagrangian does not present particular difficulties, details can be found in [1] or [3]. For convenience we introduce the constant angle $\phi_{0}=0$ (then $\dot{\phi}_{0}=\ddot{\phi}_{0}=0$ ) in such a way that the different terms write 


$$
\begin{gathered}
\mathcal{T}_{\text {pipe }}=\frac{M_{p} L^{3}}{2} \sum_{i=1}^{n}\left[\frac{\dot{\phi}_{i}^{2}}{3}+\dot{\phi}_{i} \sum_{j=0}^{i-1} \dot{\phi}_{j} \cos \left(\phi_{i}-\phi_{j}\right)+\left(\sum_{j=0}^{i-1} \dot{\phi}_{j} \cos \left(\phi_{i}-\phi_{j}\right)\right)^{2}+\left(\sum_{j=0}^{i-1} \dot{\phi}_{j} \sin \left(\phi_{i}-\phi_{j}\right)\right)^{2},\right. \\
\mathcal{T}_{\text {fluid }}=\frac{M_{f} L^{3}}{2} \sum_{i=1}^{n}\left[\frac{\dot{\phi}_{i}^{2}}{3}+\dot{\phi}_{i} \sum_{j=0}^{i-1} \dot{\phi}_{j} \cos \left(\phi_{i}-\phi_{j}\right)+\left(\sum_{j=0}^{i-1} \dot{\phi}_{j} \cos \left(\phi_{i}-\phi_{j}\right)\right)^{2}+\left(\sum_{j=0}^{i-1} \dot{\phi}_{j} \sin \left(\phi_{i}-\phi_{j}\right)\right)^{2}\right] \\
+M_{f} L^{2} U \sum_{i=1}^{n} \sum_{j=0}^{i-1} \dot{\phi}_{j} \sin \left(\phi_{i}-\phi_{j}\right)+n \frac{M_{f} L U^{2}}{2} \\
\mathcal{V}_{\text {gravity }}=-\left(M_{p}+M_{f}\right) G L^{2} \sum_{i=1}^{n}\left[\frac{\cos \phi_{i}}{2}+\sum_{j=0}^{i-1} \cos \phi_{j}\right]+\operatorname{const.}
\end{gathered}
$$

and

$$
\mathcal{V}_{\text {restor }}=\frac{K}{2} \sum_{i=1}^{n}\left(\phi_{i}-\phi_{i-1}\right)^{2}+\text { const. }
$$

The dissipation in the joints, which is neglected in the seminal study of Benjamin [1], is taken into account here through the Rayleigh dissipation function $\mathcal{R}$. The dissipative forces are supposed to be linearly dependent on the angular velocity and characterized by the damping coefficient $C$ in such a way that

$$
\mathcal{R}=\frac{C}{2} \sum_{i=1}^{n}\left(\dot{\phi}_{i}-\dot{\phi}_{i-1}\right)^{2}
$$

It should be noted that the conveyed fluid is not supposed inviscid but the system dynamics does not depend on the fluid friction because, as discussed by Benjamin [1], its overall effect on the articulated pipe is equivalent to a force parallel to the first segment, that is to $\overrightarrow{\tau_{1}}$, and therefore does not work.

Substituting Eqs. (2)-(5) into Eq. (1) we can easily derive the $n$ coupled non linear differential equations governing the motion of the articulated pipe. These equations are linearized about the equilibrium position $\left(\phi_{r}=0\right)_{r=1 . . n}$ and nondimensionalized considering the dimensionless time $t=\Omega T$ where, following Benjamin $[1], \Omega=\left(\frac{3 K}{\left(M_{p}+M_{f}\right) L^{3}}\right)^{\frac{1}{2}}$ is the frequency of the undamped $(C=0)$ oscillator made of a single horizontal $(G=0)$ segment filled with the fluid at rest $(U=0)$.

The linear nondimensional equations write, for $r=1$ to $n$,

$$
\begin{aligned}
\ddot{\phi}_{r}+\frac{3}{2}\left[\sum_{j=0}^{r-1} \ddot{\phi}_{j}+\left(1-\delta_{r n}\right) \sum_{i=r+1}^{n}\left(\ddot{\phi}_{i}+2 \sum_{j=0}^{i-1} \ddot{\phi}_{j}\right)\right] \\
+m u\left[\sum_{i=r}^{n} \dot{\phi}_{i}+\left(1-\delta_{r n}\right) \sum_{i=r+1}^{n} \dot{\phi}_{i}\right]+c\left[\dot{\phi}_{r}-\dot{\phi}_{r-1}-\left(1-\delta_{r n}\right)\left(\dot{\phi}_{r+1}-\dot{\phi}_{r}\right)\right] \\
+m u^{2}\left(\phi_{n}-\phi_{r}\right)+g[1+2(n-r)] \phi_{r}+\phi_{r}-\phi_{r-1}-\left(1-\delta_{r n}\right)\left(\phi_{r+1}-\phi_{r}\right)=0
\end{aligned}
$$

where $\delta_{r n}$ is the Kronecker delta, overdot denotes now derivative with respect to dimensionless time $t$ and the dimensionless parameters are

$$
m=\frac{3 M_{f}}{M_{p}+M_{f}}, \quad u=\frac{U}{L \Omega}, \quad c=\frac{3 C}{\left(M_{p}+M_{f}\right) L^{3} \Omega}, \quad g=\frac{3 G}{2 L \Omega^{2}} .
$$

To analyse the linear stability of the articulated pipe, we consider displacements of the segments of the form

$$
\phi_{r}(t)=\varphi_{r} e^{\sigma t}
$$



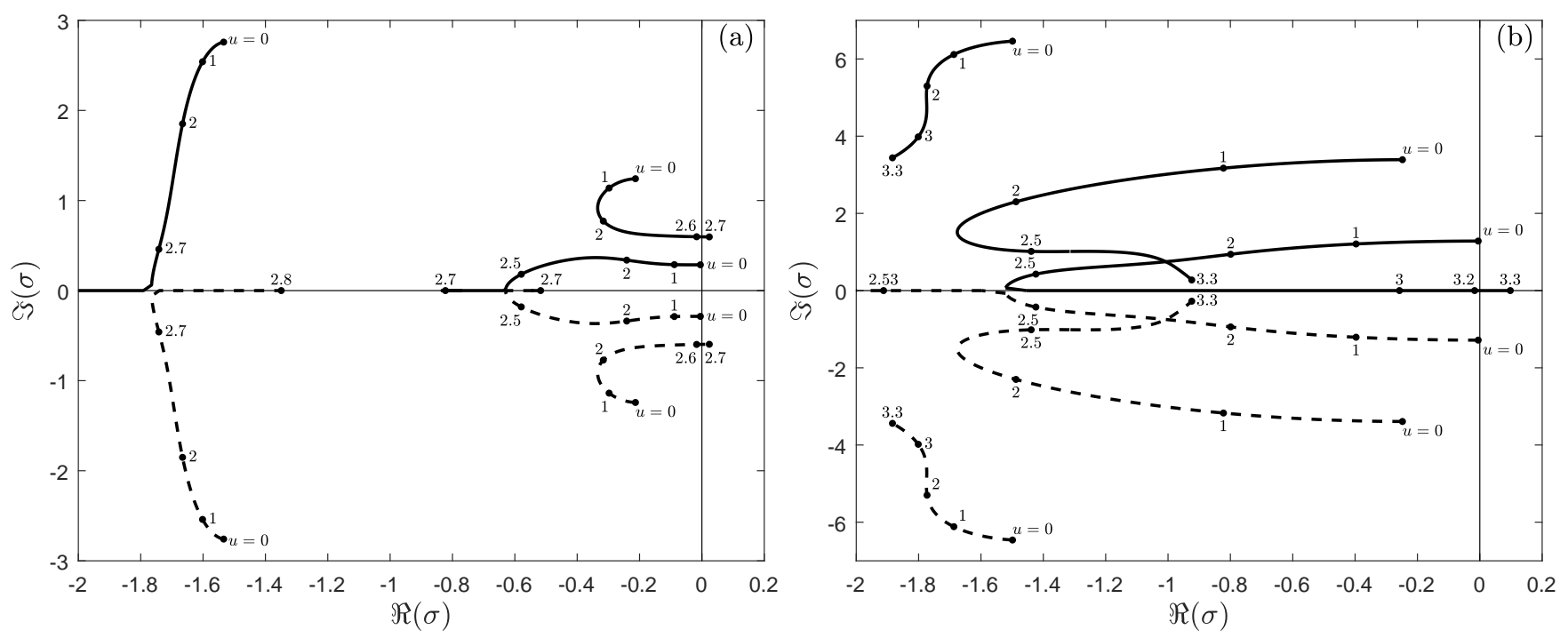

FIG. 2. Root locus diagram as the velocity $u$ is varied from 0 to the critical value $u_{c}$ for $n=3, c=0.3477$ and, (a) $m=0.4083$ and $g=0.1649$, (b) $m=2$ and $g=5$.

where the complex eigenvalue $\sigma$ is nondimensionalized with $\Omega$. By inserting in Eq. (7) we obtain a linear homogeneous system for the complex amplitude vector $\varphi=\left(\varphi_{r}\right)_{r=1 . . n}$

$$
\mathbf{a} \varphi=0
$$

where $\mathbf{a}$ is a $n \times n$ matrix. The matrix elements $a_{i, j}$, whose expressions are given in Appendix, depend on $n, m, g, c, u$ and $\sigma$. The displacement modes of the articulated pipe are the non-trivial solutions of Eq. (10) associated with $\sigma$ roots of the determinant of $\mathbf{a}$. The displacement is of buckling type, also referred to as divergence, if the circular frequency is zero $\Im(\sigma)=0$, or otherwise oscillatory or flutter. For a given configuration $(n, m, g, c)$, as $u$ is increased the static equilibrium state $\left(\phi_{r}=0\right)_{r=1 . . n}$ becomes linearly unstable as soon as one of the displacement modes has a positive growth rate $\Re(\sigma)>0$. The critical velocity $u_{c}$ is given by $\Re(\sigma)=0$.

Figure 2 shows the locus of the roots $\sigma$ on the complex plane for an articulated pipe made of $n=3$ segments as $u$ is varied from 0 to just above the critical velocity $u_{c}$ for two different configurations. In Fig. 2(a) the values of the parameters correspond to the present experiments $(m=0.4083, g=0.1649, c=0.3477)$ and the hanging equilibrium position loses stability by flutter at $2.6<u_{c}<2.7$ where a pair of complex roots cross the imaginary axis. For the configuration of Fig. 2(b), where $m=2, g=5$ and $c=0.3477$, a single purely real root cross the imaginary axis, at $u_{c}$ between 2.1 and 2.2 , that is indicative of divergence.

\section{RESULTS}

The experimental results obtained for articulated pipes made of $n=2$ to 5 segments are presented and compared to the predictions of the linear stability analysis performed with the experimental values of the dimensionless parameters, namely $m=0.4083, g=0.1649$ and $c=0.3477$.

As shown in the previous section, when $u$ is increased the destabilisation of the freely hanging articulated pipe can give rise to divergence- or flutter-type instability depending on the value of the parameters $n, m, g$ and $c$. The boundary which separates the regions where the articulated pipe loses stability by flutter or by divergence, as calulated with the linear model, is plotted in Fig. 3 in the plane $(g, m)$ for the experimental value $c=0.3477$ and for $n=2,3$, 4 and 8. Flutter appears to be confined to the region of low values of $g$ or $m$ and from $n=4$ all curves almost overlap for a given $c$. The boundaries calculated for $n=2$ segments for joints without damping $c=0$ and for $c=1$ are also plotted to highlight that the effect of increasing $c$ is to reduce the region of flutter. Note that the curve for $c=0$ and $n=2$ has already been presented by Lunn (reproduced in [10]). The points labeled (a) and (b) in Fig. 3 correspond to the conditions of Fig. 2 (a) and (b), respectively. Point (a) represents the present experimental conditions $m=0.4083$ and $g=0.1649$ and, in agreement with our observations where loss of stability only occurs by flutter, this point is clearly in the flutter region whatever the number $n$ of segments. 


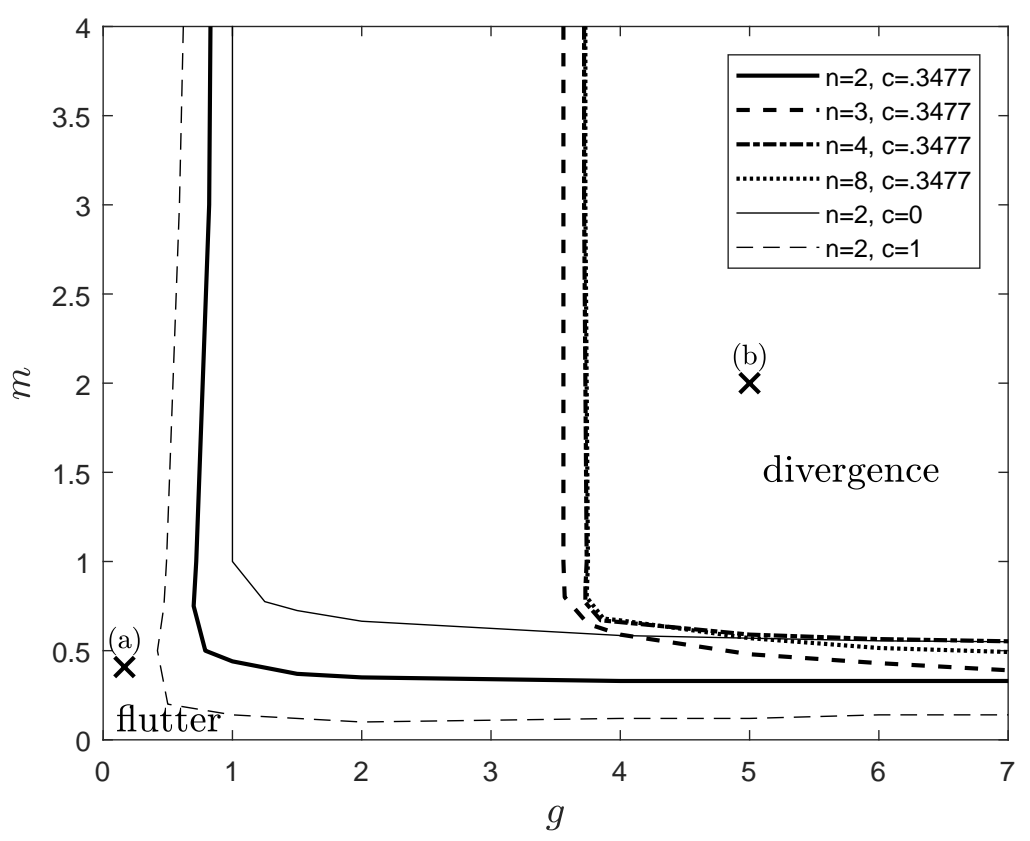

FIG. 3. Boundaries between regions of loss of stability of the freely hanging articulated pipe by flutter and divergence for $c=0.3477$ and $n=2,3,5$ and 10 , and for $c=0$ and 1 and $n=2$. The points labeled (a) and (b) correspond to the conditions of Fig. 2(a) and (b), respectively.

The stability of a hanging articulated pipe is experimentally investigated by gradually increasing the flow velocity $U$. The behavior appears qualitatively similar for all the pipes and to the observations in [2] for two-segment pipes. Without flow $(U=0)$, as the pipe is perturbed it returns to its equilibrium position after a few decaying oscillations. At low velocities, the sytem is overdamped that is the pipe settles back to the equilibrium without oscillations, while, when $U$ is further increased, oscillations occur which decay over a time increasing with $U$. Then above a critical value $U_{c}$ of the flow, the oscillations grow to reach a limit cycle of frequency $F_{c}$, corresponding to the flutter instability. The experimental dimensionless critical velocity $u_{c}=U_{c} /(L \Omega)$ and circular frequency $\omega_{c}=2 \pi F_{c} / \Omega$ are plotted in Fig. 4(a) and (b), respectively, as function of $n$. For each $n$, the experiment is repeated several times. If the same pipe is used, the same critical velocity and frequency are found within the experimental uncertainty. In contrast, if different pipes are used, that is a pipe made with new joints and segments randomly changed, dispersion of the experimental results can reach almost $10 \%$ for both quantities. For example for $n=2$ critical velocities are found between 11.5 and $12.3 \mathrm{~m} \cdot \mathrm{s}^{-1}\left(2.75 \leqslant u_{c} \leqslant 2.95\right)$ and critical frequencies $1.94 \leqslant F_{c} \leqslant 2.08 \mathrm{~s}^{-1}\left(0.57 \leqslant \omega_{c} \leqslant 0.61\right)$. This dispersion is represented by error bars in Fig. 4. It is believed that this dispersion is the result of the dispersion of the measurements of the stiffness $K$ and damping $C$ coefficients for different joints mentionned in §II.

The behavior of the articulated pipes as $u$ is increased can be interpreted by the analytical results previously presented, by looking at the trajectory of all the eigenvalues $\sigma$ in the complex plane. Considering the example of the Fig. 2(a) where $n=3$ (but it is similar whatever $n$ ) we see that all the eigenvalues move first towards smaller negative values of the growth rate $\Re(\sigma)$ then at least a pair of eigenvalues goes in the other direction up to get a positive growth rate at a critical value $u_{c}$. The critical velocity and frequency predicted by the linear analysis are also plotted in Fig. 4(a) and (b) for comparison; the analytical critical frequency is the value of $\Im(\sigma)$ at $u_{c}$. For the small range $2 \leqslant n \leqslant 5$ considered during the present experiments, the decrease of $u_{c}$ and $\omega_{c}$ with $n$ is qualitatively well described and quantitatively not very far from the experimental values although overestimated for $n=2$ and 3 .

The analytical results for the critical velocity $u_{c}$ of Fig. 4(a) reveal ranges in $n$ with a local minimum. These ranges correspond to flutter modes of a different structure illustrated by the superimposed views of the pipe during one flutter period in the insets of the figure for $n=4,8$ and 16. For pipes made of $n=7$ to 12 segments, the flutter mode has one node (marked by the arrow) and for larger $n$, mode with two nodes, and possibly more nodes for still more segments, are predicted. For shortest pipes $(2 \leqslant n \leqslant 6)$, as considered in the experiments, flutter modes with zero node are obtained at the threshold.

This is confirmed in Fig. 5 where the shape of the flutter modes observed during the experiments at the threshold is illustrated by superimposing views of the pipe during one flutter cycle, the flutter modes predicted by the linear stability analysis are also shown. Comparison reveals two main differences.

First, contrary to the analytical futter modes, the experimental ones present a more or less pronounced asymmetry 

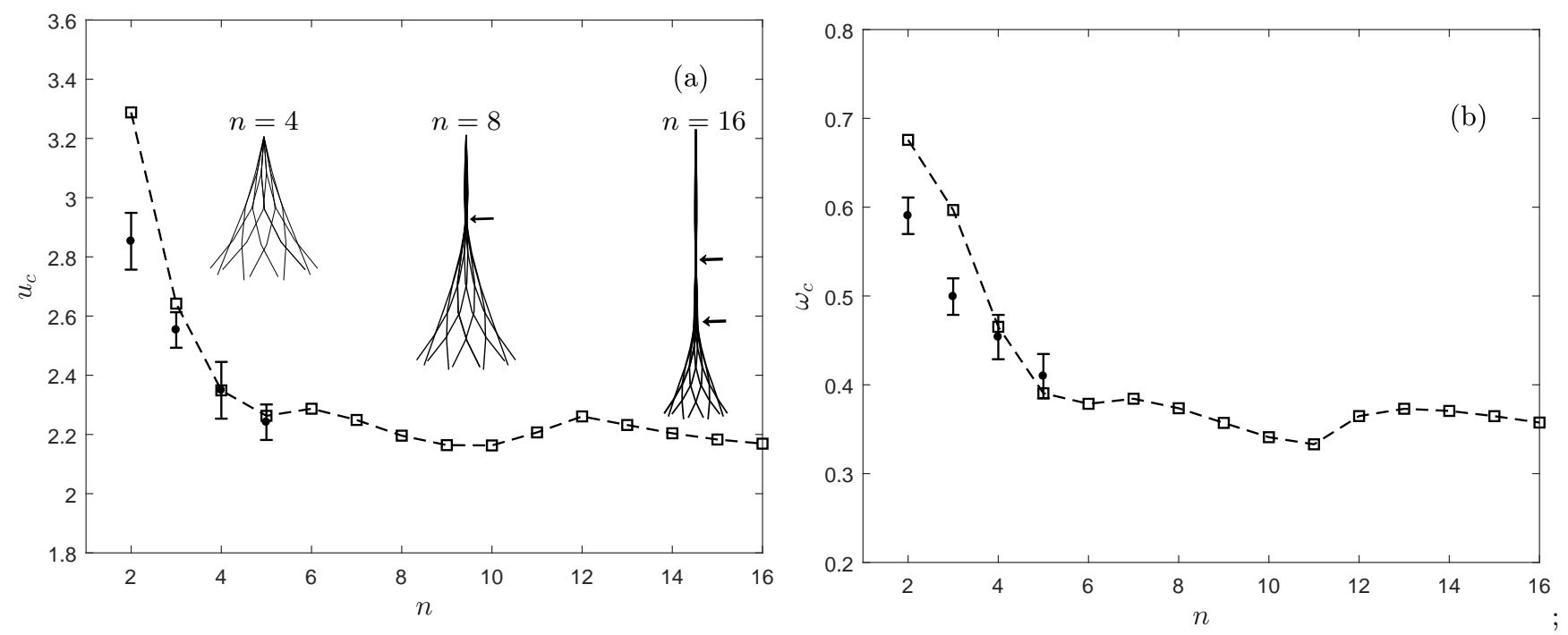

FIG. 4. Dimensionless critical velocity $u_{c}$ (a) and circular frequancy $\omega_{c}$ (b) as function of the number of segments $n$ as predicted by the linear stability analysis $(\square)$ and as obtained during the experiments $(\bullet)$, the error bars represent the experimental value dispersion. The shape of the flutter modes at the threshold calculated for $n=4,8$ and 16 are shown.

with respect to the vertical axis. This asymmetry seems to be reminiscent of an assymmetry of the equilibrium states, indeed, the hanging articulated pipe without flow is inevitably not perfectly straight in the experiments in such a way that the flow gives rise to lateral forces leading to the increase of the alignment defect at the joints, as already mentionned by Benjamin [2]. We illustrate this behavior choosing a configuration $(n=3$ in Fig. 5$)$ for which the flutter is strongly asymmetric and we present in Fig. 6 the static equilibrium states that precede this flutter for different flow velocity $u<u_{c}$. The pipe is seen to be more and more deflected from the vertical straight position as $u$ is increased and the flutter mode is shifted in the same direction as the preceding static equilibrium states.

Another difference we can note by comparing experimental and analytical mode shapes is the distribution of the flutter amplitude of each segments along the pipe. The amplitudes predicted by linear stability analysis being arbitrary, we compare in Fig. 7 the angular amplitudes $\Phi_{r}$ of the oscillatory motion of each segment $r$ normalized by the amplitude of the last segment of the pipe $\Phi_{n}$. Experimental values in Fig. 7 correspond to the flutter motions of Fig. 5, the dispersion of these quantities as the experiments are repeated is small of the order of the symbol size. For all $n$, the model predicts a gradual increase of the amplitude from the first (highest) segment to the last (lowest). In contrast, the experiments show a flutter amplitude more and more confined to the last segments for $n$ above 2 and the amplitude of a segment appears smaller and smaller, when compared to the analytical results, as the number of segments below it increases.

This discrepancy is due to assumption made in the analysis that all the joints are identical. Indeed, damping in a joint comes on one hand from the dissipation in the joint material and, on the other hand, from the friction of the segments on the axis of the pivot link. The latter depends on the force applied on the axis that is mainly due to the weight and to the fluid friction. Both effects increasing with the number of segments below the joint. It results that the higher the segment, the larger the damping coefficient $C$ and $C$ is maximum for the highest joint. In contrast in the model $C$ is supposed to be constant and equal to the mean value experimentally measured for a single segment without flow (see $\S \mathrm{II})$.

\section{CONCLUDING DISCUSSION}

Experiments have been conducted to investigate the stability of articulated pipes conveying a fluid as the flow velocity is increased. For the value of the experimental parameters considered in the present study, the pipes lost stability by flutter. The critical velocities have been measured and the resulting flutter modes have been characterized for a number of segments $n$ between 2 to 5 . The configuration with only one segment $(n=1)$ is not discussed because its equilibrium position $\left(\phi_{1}=0\right)$ is always stable as experimentally observed and confirmed by the linear analysis. This can be understood by considering the energy transfer from the fluid flow to the pipe that writes over a time $\Delta T$ 


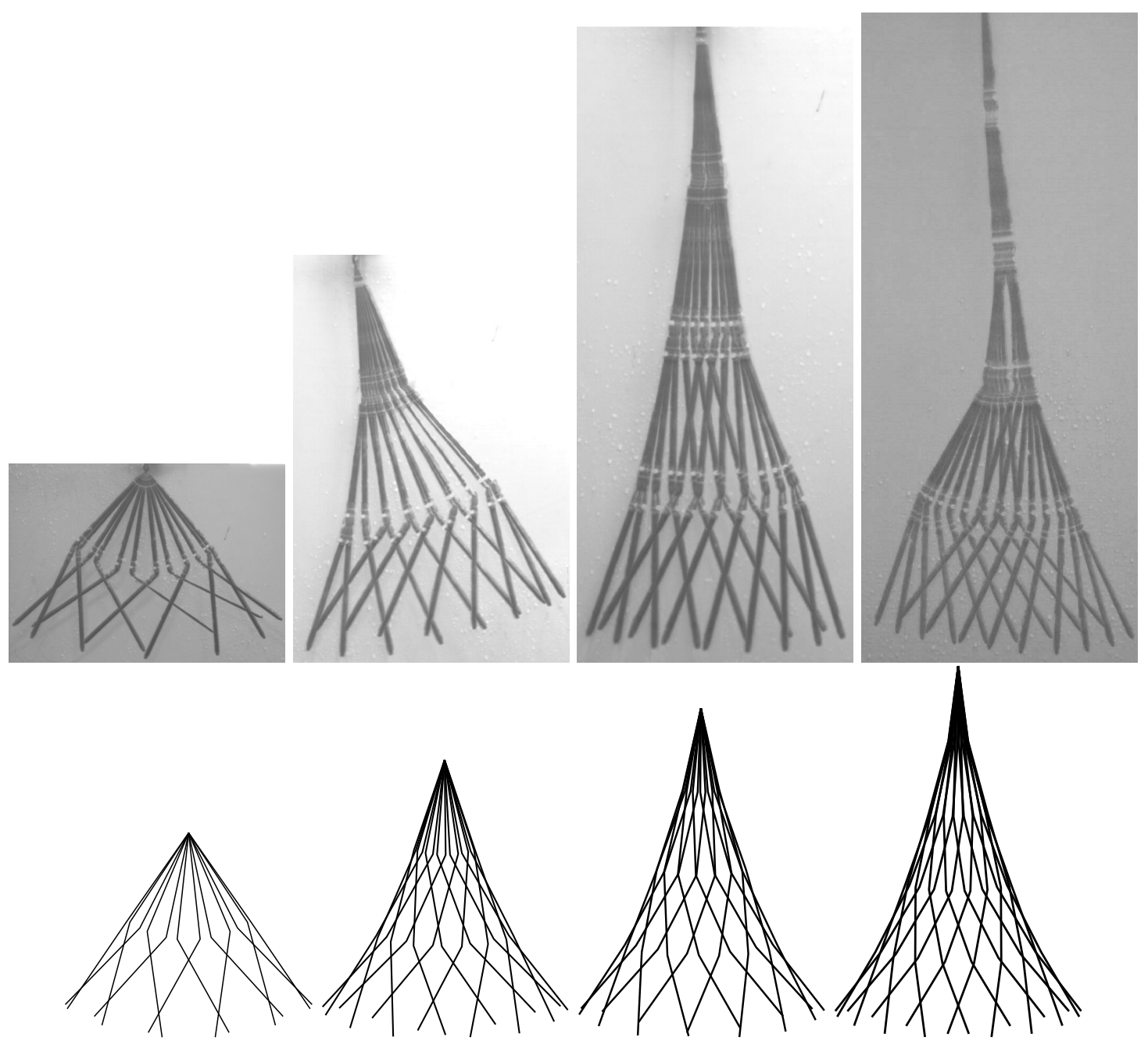

FIG. 5. Superimposed views of the articulated pipe during one flutter cycle at the threshold for $n=2,3,4$ and 5 (from left to right) as recorded during the experiments (top) and predicted by the linear analysis (bottom).

$($ see $[1])$

$$
\Delta W=-\int_{T}^{T+\Delta T} M_{f} U\left(\dot{O E}^{2}+U \overrightarrow{\tau_{n}} \cdot \dot{\overrightarrow{O E}}\right) \mathrm{d} T
$$

The first term in the integrand is always positive and the second term is zero when $n=1$, it results that $\Delta W$ is always negative for the configuration with only one segment in such a way that flutter can not occur. Configurations comprising of more segments were initially going to be considered but, due to the increase of the head loss with the pipe length, during all the attempts with $n=6$ segments we get a fast swelling then explosion of the first joint. This occured sometimes during the experiments with 5 segments.

Linear stability analysis of an idealized system has also been performed extending previous works. Comparisons with the experimental results have shown the ability of the model to describe at least qualitatively the flutter instability at the threslold. But two complementary experimental observations must be mentionned. First, the critical velocity $u_{c}$ for the occurence of flutter has been experimentally determined by increasing flow velocity. If the velocity was then decreased the flutter persisted up to $u_{d} \leqslant u_{c}$. These hysteresis loops, already reported in [2], could be also reponsible for the dispersion of the experimental values of $u_{c}$ in Fig. 4(a), their wideness defined as $\left(u_{c}-u_{d}\right) / u_{c}$ have been found to be smaller than $9 \%$ for all pipes. Second, for increasing as well as for decreasing velocities only finite amplitude flutter has been observed, in other words flutter such that $\Phi_{r} \ll 1$ for all $r=1$ to $n$ has never been observed. These two points suggests that to go further than the characterization of the flutter at the threshold it would be necessary to consider the system of nonlinear equations governing the pipe dynamics and obtained by substituting Eqs. (2)-(5) 


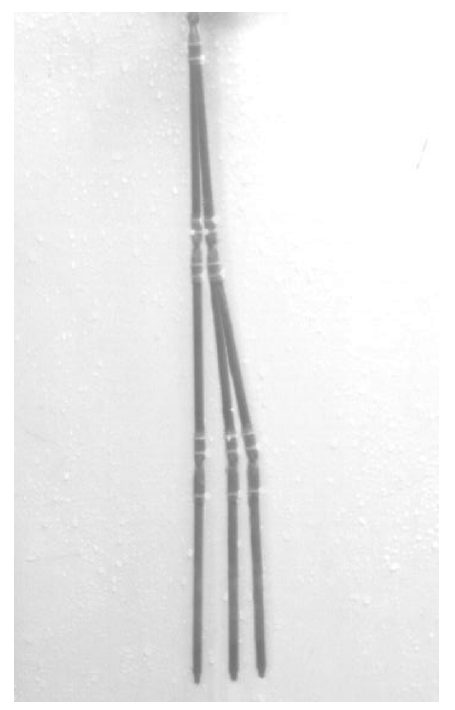

FIG. 6. Superimposed views of the equilibrium states for $U=0,6.8$ and $7.8 \mathrm{~m} . \mathrm{s}^{-1}(u=0,1.63$ and 1.87 from left to right) preceding the flutter shown in Fig. 5 for $n=3$.
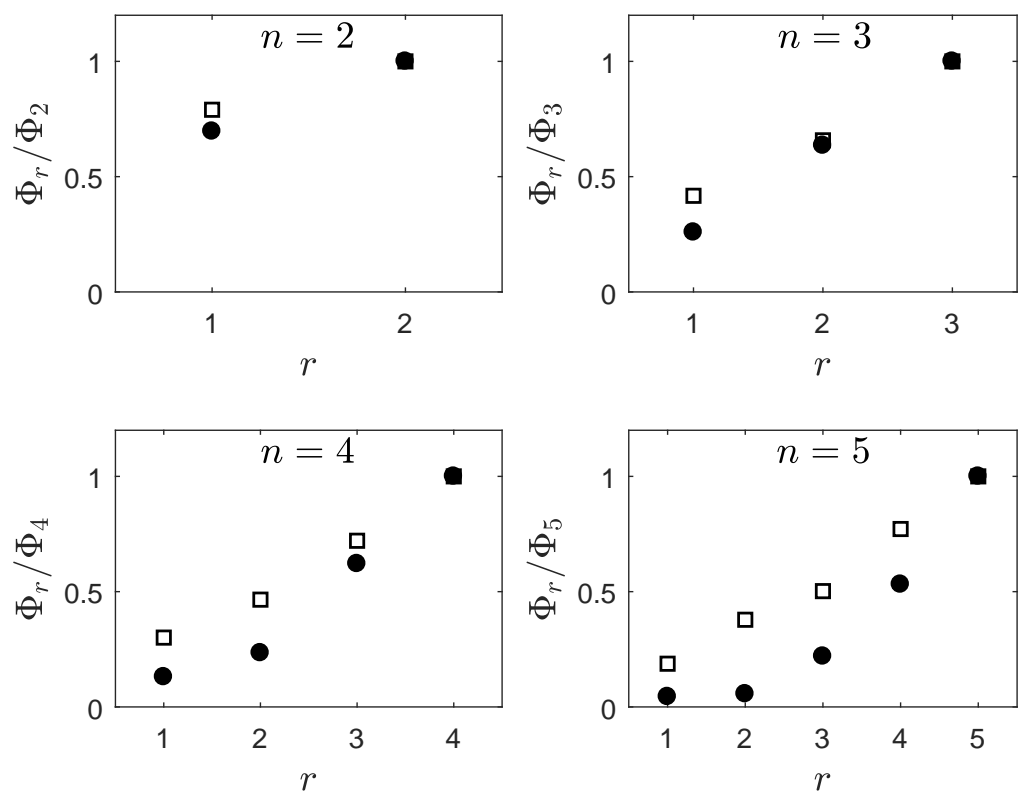

FIG. 7. Angular amplitude of the motion of the segment $r$ normalized by the amplitude of the last segment of the pipe, for the experimental $(\bullet)$ and analytical $(\square)$ flutter modes shown in Fig. 5.

into Eq. (1). Investigations of the nonlinear dynamics of articulated pipes are currently underway both experimentally and numerically integrating the resulting system of ordinary differential equations using a Runge-Kutta method. 


\section{Appendix}

The elements of the $n \times n$ matrix a (where $n$ is the number of segments into the pipe) of the linear system Eq. (10) depend on the dimensionless quantities $m, g, c, u$ and $\sigma$, themselves defined as functions of the physical parameters of the system in §III. Expressions of these elements are given hereafter:

For all $n$

$$
\begin{gathered}
a_{n, n}=\sigma^{2}+(m u+c) \sigma+g+1, \\
a_{n-1, n}=\frac{3}{2} \sigma^{2}+(2 m u-c) \sigma+m u^{2}-1,
\end{gathered}
$$

and for $i=1$ to $n-1$

$$
\begin{gathered}
a_{i, i}=[4+3(n-1-i)] \sigma^{2}+(m u+2 c) \sigma-m u^{2}+[3+2(n-1-i)] g+2, \\
a_{i+1, i}=[3+6(n-1-i)] \frac{\sigma^{2}}{2}-c \sigma-1 .
\end{gathered}
$$

If $n>2$ we have for $i=1$ to $n-2$

$$
\begin{gathered}
a_{i, n}=\frac{3}{2} \sigma^{2}+2 m u \sigma+m u^{2}, \\
a_{i, i+1}=[9+6(n-2-i)] \frac{\sigma^{2}}{2}+(2 m u-c) \sigma-1,
\end{gathered}
$$

and for $j=1$ to $i$

$$
a_{i+2, j}=[3+6(n-2-i)] \frac{\sigma^{2}}{2} .
$$

If $n>3$ we have for $i=1$ to $n-3$ and $j=i+2$ to $n-1$

$$
a_{i, j}=[9+6(n-1-j)] \frac{\sigma^{2}}{2}+2 m u \sigma .
$$

[1] T.B. Benjamin, "Dynamics of a system of articulated pipes conveying fluid. I. Theory," Proc. Roy. Soc. A 261, 457 (1961).

[2] T.B. Benjamin, "Dynamics of a system of articulated pipes conveying fluid. I. Experiments," Proc. Roy. Soc. A 261, 487 (1961).

[3] M.P. Païdoussis, E.B. Deksnis, "Articulated models of cantilevers conveying fluid: The study of a paradox," J. Mech. Engng. Sci. 12, 288 (1970).

[4] M.P. Païdoussis, "Dynamics of tubular cantilevers conveying fluid," J. Mech. Engng. Sci. 12, 85 (1970).

[5] J. Rousselet, G. Herrmann, "Flutter of articulated pipes at finite amplitude," J. Appl. Mech. 44, 154 (1977).

[6] P.R. Sethna, S.W. Shaw, "On codimension-three bifurcations in the motion of articulated tubes conveying fluid," Physica D 24, 305 (1987).

[7] M.P. Bohn, G. Herrmann, "Instabilities of a spatial system of articulated pipes conveying fluid," J. Fluids Eng. 96, 289 (1974).

[8] M.P. Bohn, G. Herrmann, "The dynamic behavior of articulated pipes conveying fluid with periodic flow rate," J. Appl. Mech. 41, 55 (1974).

[9] J.S. Jensen, "Articulated pipes conveying fluid with high frequency," Nonlinear Dyn. 19, 171 (1999).

[10] M.P. Païdoussis, Fluid-Structure Interactions. Slender Structures and Axial Flow, volume 1 (Academic Press, San Diego, 1998). 